\title{
Research on China's Health Industry Governance System Based on Synergy Theory
}

\author{
Lei Chen ${ }^{1}$ Hongyang Shi ${ }^{2, *}$
}

\author{
${ }^{1}$ Jiangxi University of Traditional Chinese Medicine, Nanchang, Jiangxi, China \\ ${ }^{2}$ Jiangxi University of Traditional Chinese Medicine, Nanchang, Jiangxi, China \\ *Corresponding author.Email: ecoshy@qq.com
}

\begin{abstract}
A modern health industry governance system is the key to the high-quality development of the health industry and helps realize people's yearning for a better life. Based on the theory of synergy, this paper constructs a modern governance system for the health industry. It suggest that the modern governance system of the health industry should include market coordination, regulatory coordination, social coordination, and cultural coordination. The results show that: China's health industry has insufficient resource input, insufficient regional allocation, and industrial institutions that need to be further improved; lack of regulatory coordination, and it is urgent to improve the internal and external coordination and supervision mechanism; lack of talents leads to problems such as insufficient innovation capabilities. Therefore, it is necessary to establish an open, efficient and standardized market system; strengthen the coordination of regulatory mechanisms; strengthen health education, guide the change of social concepts, establish a modern governance system for the health industry that is governed by the government, enterprises, and society, and strengthen the construction of new infrastructure so that the development of the health industry can meet the needs of the people and the diversity of life.
\end{abstract}

Keywords: Health industry, health industry develop, health industry governance system

\section{INTRODUCTION}

Since the founding of the People's Republic of China, improving the health of the people has always been the core of government's healthy policy. The government attached great importance to improving our health. In the 1950 s, China's health work focused on rural areas. From the 1980s to the early 1990s China's health work policy clearly states that "relying on scientific and technological progress to serve the people's health" has laid the basic framework for China's healthy work. In 2009, the "Opinions of the Central Committee of the Communist Party of China and the State Council on Deepening the Reform of the Medical and Health System" proposed to build a four-in-one basic medical and health system covering urban and rural areas to further deepen the connotation of the health work policy. In 2016, the underlying logic of health policy changed: the National Health and Sanitation Conference held in Beijing in August 2016 formally proposed the concept of "big health", marking China Health work "shifts from diseasecentered to health-centered". On October 25, 2016, in accordance with the strategic deployment of the Fifth
Plenary Session of the 18th National Congress of the Communist Party of China, the State Council issued the "Healthy China 2030" Planning Outline, putting forward the theme of "Jointly Building and Sharing, Health for All", and requested that by 2030 , promote The system of health for all has been improved, the development of the health field has been more coordinated, health lifestyles have been popularized, the quality of health services and health protection levels have been continuously improved, the health industry has prospered, and health equity has basically been realized, and the main health indicators have entered the ranks of high-income countries $^{[1]}$. With the development of China's economy and society and the improvement of people's living standards, the people's yearning for a better life has been given more and new content, especially "accelerating the formation of a domestic and international double cycle that promotes each other. Under the new development pattern of China, a more adequate, balanced, and higherquality health industry development is the guarantee for realizing the people's needs for a better life. Therefore, the prerequisite for achieving "co-construction and sharing, health for all" is the high-quality development of 
health industry, this requires that the health industry must meet the differences in the health needs of the people and the diversity of life.

\section{THE KEY TO THE HIGH-QUALITY DEVELOPMENT OF THE HEALTH INDUSTRY}

The high-quality development of the health industry should reflect the development concept of "innovation, coordination, green, openness, and sharing" and meet the new and higher health needs of the people. The modern governance system covers multiple fields such as economy, politics, culture, society, and ecological civilization. It is an institutional mechanism formed by closely intertwining and coordinating each field. The health industry covers various fields such as medicine, medical treatment, medical insurance, health products, health food, health management, beauty and health, health information, health culture, etc., forming a relatively complete industrial chain, but due to the particularity of its products and services, Prone to problems such as cross-formats and the edge of the supervision system ${ }^{[2]}$. At present, academic research on the development of the health industry has not yet formed a corresponding theoretical basis. This article conducts research and analysis on the development of the health industry based on the theory of synergy, and provides reference opinions for promoting the high-quality development of the health industry. The purpose of the study of synergy theory is to deal with the problems of complex systems by constructing a systematic viewpoint and propose solutions ${ }^{[3]}$.

The theory of synergy has a high degree of compatibility with health industry governance. Health industry governance is a systematic process involving multiple subjects and multiple departments, emphasizing the cooperation and coordination of multiple subjects. The theory of synergy is the science of dealing with the competition and cooperation of various subsystems in the system, and it is very suitable for dealing with the mutisubject cooperation and synergy of health industry governance. From the perspective of synergy theory, the current health industry governance system in China has the characteristics of complexity, openness, internal nonlinear effects, and far from equilibrium. The specific manifestations are as follows: First, the health industry governance system is a complex system. Each component is non-linear and random, and interacts with the environment and influences each other. Second, the health industry governance system is a dynamic and open system. To continuously output crisis management functions, the system needs to continuously exchange various elements with the outside world. Third, the health industry governance system has a non-linear effect inside. The health industry governance involves multiple factors, and the connection is complex, and there is a non-linear effect. Fourth, the health industry governance system is in an unstable state.

Therefore, the key core to promote the high-quality development of the health industry is the collaborative governance of the health industry, which is mainly reflected in the four aspects of market coordination, regulatory coordination, social coordination, and cultural coordination.

\subsection{Market Synergy, An Important Foundation for Collaborative Governance In the Health Industry}

The formation of an open, standardized and unified market system is conducive to the free flow of factors between regions, which not only significantly improves the labor productivity of the regions where the factors flow into, but also effectively increases the factor return rate of the regions where the factors flow out, showing a trend of coordinated agglomeration ${ }^{[4]}$, reduce transaction costs. The requirements of market coordination for the high-quality development of the health industry: First, guide and realize the independent and orderly flow of labor, capital, technology and other elements and the free trade of resources. Second, form an open, standardized and unified market system, and create a fair competition and orderly market environment. Third, we must further optimize the industrial structure, optimize the reallocation of resources, and promote the quality and upgrade of health services.

\subsection{Supervision Synergy, An Important Guarantee for the Collaborative Governance of the Health Industry}

The health industry covers the primary, secondary, and tertiary industries, encompassing multiple fields, and its products and services show the characteristics of multiple structural levels. The supervision involves the State Administration of Market Supervision, the National Health Commission, and the State Food and Drug Administration. In the complex situation of the crossformat of the health industry, regulatory marginal issues are extremely prone to appear, especially in the fields of medicines and health care products. The inherent requirements of supervision coordination in the highquality development of the health industry are: First, it is necessary to improve the supervision and management mechanism and establish an efficient and unified supervision and management system. Second, enterprises must build an internal supervision mechanism, reduce costs, and promote development through independent governance. Third, it is necessary to clarify the marginal areas of supervision, further improve the supervision mechanism of health products such as drugs and health products, and strengthen the safety of health products. 


\subsection{Social Synergy, An Important Support for the High-quality Development of the Health Industry}

The products and services provided by the health industry have a certain degree of supplier dominance and a certain degree of information asymmetry, which easily leads to moral hazard and adverse selection, and seriously hinders the high-quality development of the health industry. Social collaboration requires the health industry to have a certain degree of flexibility and adaptability, focus on new growth points in consumer demand, and continue to meet the diversified, individualized, and differentiated health needs of the society. By integrating social resources, the government, market, and The cooperative governance of society and the public promotes the high-quality development of the health industry. This requirement: On the one hand, it is necessary to establish and improve an information sharing mechanism, reduce information asymmetry, build trust between enterprises and consumers, and accept consumer supervision. On the other hand, it is necessary to establish and improve the demand collection and feedback mechanism, focus on the increasingly diverse health needs, and effectively meet the growing new demands.

\subsection{Cultural Synergy, The Source of Innovation for the High-quality Development of the Health Industry}

China's health industry is mainly based on the provision of traditional medical services, which cannot effectively cover the people's needs for "prevention of disease" such as health preservation and health care. The "Healthy China" strategy has pointed out the direction for the development of China's health industry. Strengthening the in-depth integration of the health industry and traditional Chinese medicine is conducive to promoting the innovative development of the health industry, improving the quality of labor, and gathering talents; it is conducive to promoting the optimal allocation of various resources, fostering market advantages, and promoting the high-quality development of the health industry. This requires: First, we must establish new public cultural communication channels, give full play to the advantages of the latest information technology and new media resources, and promote the dissemination of Chinese medicine culture. Second, strengthen the construction of health innovation industrial parks, enhance the concentration of the health industry, innovate health products, and provide a good environment for talents. Third, it is necessary to optimize talent training channels to adapt to the popularization of higher education.

In summary, the modern health industry quality system includes market coordination, regulatory coordination, social coordination, and cultural coordination governance. The high-quality development of the health industry cannot be separated from the modern health industry governance system. The highquality development of the health industry can help realize people The masses' yearning for a better life.

\section{PROBLEMS IN THE DEVELOPMENT OF CHINA'S HEALTH INDUSTRY}

\subsection{Insufficient Resource Input, Insufficient Regional Allocation, and Industrial Structure to Be Optimized}

First, insufficient investment in health funds has led to an overall shortage of health resources. According to statistics, the total global annual medical and health expenditure accounts for $10 \%$ of the world's gross product (GWP). In 2015, China's total medical and health expenditures accounted for $4.89 \%$ of GDP. In 2016, the ratio was $4.98 \%$. In 2017 , the ratio was $5.15 \%$. Although China's total medical and health expenditures as a percentage of GDP are increasing year by year, the growth rate has been slow. 123rd in the world. In 2017, the total expenditure on health care in the United States accounted for $17.06 \%$ of GDP, France accounted for $11.31 \%$, Japan accounted for $10.96 \%$, the Russian Federation accounted for $5.34 \%$, and Vietnam accounted for $5.53 \%$ [Data source: World Health Organization Global Health Data Observatory ]. Insufficient investment in health funds has led to an overall shortage of health resources in China.

Second, regional development is not balanced. China's high-quality health-care resources and innovation resources are mainly concentrated in developed cities or provincial capitals such as Beijing, Shanghai and Guangzhou. Primary medical institutions have problems such as small scale, poor service quality, and insufficient information sharing among medical institutions. Restrict the high-quality development of China's health industry. Third, the optimization of the industrial structure is unreasonable, and there are structural differences between the content of health supply and the needs of the main body. At present, China's health industry chain is basically formed, but the level of the industry chain is low, some links are weak, and the innovation chain is insufficient, mainly reflected in the problem of insufficient development. The complete health industry chain should cover all areas of prevention, diagnosis and treatment, maintenance and improvement of health. The vast majority of resources in China's health-care industry are concentrated in traditional medical services, while investment in preventive healthcare is very small. Health education, health risk assessment, etc. still need to be further improved, unable to provide consumers with comprehensive and multilevel health services. China is rapidly aging, and the 
development process of the health industry is far from being able to adapt to this process.

\subsection{The Relevant Supervision and Management System Needs to Be Further Improved, and the External Supervision and Internal Supervision Coordination Mechanism of the Health Industry Will Be Improved}

First, the health industry covers the primary, secondary, and tertiary industries. Although there have been specialized regulatory departments for various industries, the coordination between the various departments is poor, and a coordinated whole has not yet been formed. According to the "China Food and Drug Administration Statistics The data from the Yearbook shows that the current gap in supervisory personnel in China is relatively large, the supervisory power is relatively weak, and the configuration of technical equipment is still insufficient[5], making supervision difficult. The second drug regulatory mechanism needs to be further improved. At present, there are many shortcomings and deficiencies in drug supervision in the access, quality, and circulation links, and there are various problems such as "multiple names of the same drug" and "inflated pricing". In recent years, the government has adopted a variety of methods such as centralized procurement and other methods to improve the comprehensive management of drugs. Although it has achieved initial results, it has not fundamentally established a mechanism to promote sustainable and healthy development of drug production and circulation. The production and circulation approval mechanism still needs to be improved. At the same time, other related supervision such as special medical foods, functional foods, and dual-purpose Chinese medicinal materials other than medicines also need to be further optimized and perfected. Third, the internal control mechanism of the health industry is imperfect, and external information sharing is not transparent. In an environment of information asymmetry, consumers cannot grasp the cost and quality of a company's products or services in advance, and companies generally have problems such as low creditworthiness and high prices of products or services, which severely restrict the high-quality development of the health industry.

\subsection{Insufficient talents and lack of innovation capabilities, and the health product innovation chain has not yet effectively formed}

First, the lack of talents restricts the high-quality development of the health industry. At present, the cultivation of talents in the health industry in China is still stuck in the barriers of elite higher education and cannot adapt to the popularization of higher education, especially the overall situation of the people's yearning for a better life. The limited medical talents and even the broader health industry's human resource allocation is seriously unreasonable and structurally unbalanced. The new crown epidemic and related public health emergencies have seriously exposed the problem of insufficient health human resources in China. Second, insufficient attention is paid to personnel training. Although the number of applicants for health management personnel has increased in recent years, it still cannot meet the market's demand for talents in the health industry, and is far from meeting the needs of the people for preventive health and other health needs. The training and education system of the health industry has not been fully formed, and it is difficult to establish a high-end health industry service team. At present, the vast majority of employees in the health industry in China still have not received professional skills training and systematic education, and they cannot satisfy both quality and quantity. The needs of China's health industry development. Third, the capacity for independent innovation is insufficient. China's health industry companies are generally small in scale, and their innovation capabilities in health technology fields such as medical technology and biotechnology are weak. They are more application innovation and imitative innovation, and industrial chain innovation based on industrial development is far from adequate.

\section{SOME SUGGESTIONS FOR THE DEVELOPMENT OF HEALTH INDUSTRY}

\subsection{Optimize the Business Environment of the Health Industry and Fully Respect the Laws of the Market}

An open, efficient and standardized market system is an important foundation for the high-quality development of the health industry. First, we must optimize the market allocation of integrated factors. Establish a more complete health industry factor market, enrich factor resources, continuously optimize the allocation of funds, labor, innovation and other resources among different institutions and departments, and effectively solve the problem of regional imbalances in resource allocation. Second, speed up the construction of regional characteristic health industries. It is necessary to give full play to the comparative advantages of the health industry in different regions, establish a regional development path tailored to local conditions, and create a regionally characteristic health industry. Third, the government should increase financial investment, improve the integrated construction of infrastructure, and guide social capital to tilt toward the health industry. Insufficient investment in health funds has resulted in an overall shortage of health resources in China and hindered the development of China's health industry. Fourth, raise the barriers to entry in the health industry market. It is necessary to strictly review the qualifications 
of health industry practitioners, improve industry access, and further regulate the health industry market environment.

\subsection{Strengthen the Coordination of Regulatory Mechanisms and Promote the Formation of External Supervision and Internal Independent Governance of the Health Industry}

The muti-department coordination and supervision mechanism is an important guarantee for the high-quality development of the health industry, and the social governance system of multiple co-governance is an important support for the high-quality development of the health industry. First, we must improve the coordination efficiency of government organizations. Health industry products involve a wide range of fields, many regulatory agencies, and management coordination is difficult. It is necessary to pay attention to the communication and coordination between multiple entities and multiple departments, and effectively promote the coordinated supervision of the health industry by multiple entities through cross-field and cross-department collaboration. Second, we must carry out third-party certification for the health service industry. Promote enterprises to adopt advanced quality management models and methods to disclose product and service quality information. Establish a third-party certification body for the quality of health industry products and services, regularly conduct quality evaluations of the health industry, disclose the evaluation results to the public, and order rectification of violating companies. Third, it is necessary to establish a health industry associations and formulate industry association regulations. Strengthen internal self-discipline in the health industry and promote independent governance from within the industry. Through government governance, social governance, and enterprise selfgovernment, we will create a new pattern of modern governance in the health industry.

\subsection{Strengthen health education and promote the culture of TCM health preservation}

The concept of life affects behavior and lifestyle. Therefore, it is necessary to promote the concept of healthy life in the whole society, strengthen health education, integrate health education into the national education system, popularize health knowledge, guide the people to establish a positive outlook on health, promote the culture of traditional Chinese medicine, and make Chinese medicine "curative" The concept of not getting sick is deeply rooted in the hearts of the people. First, innovate propaganda methods. The development of Internet technology has enabled information to break through the limitations of time and space and spread more efficiently. Therefore, to give full play to the role of the Internet and other emerging media in health education, national and local mainstream media must strengthen public opinion guidance and run popular health science programs that the people like to see. Second, we must give full play to the important role of Chinese medicine in health care, promote the culture of traditional Chinese medicine, and give full play to the unique advantages of traditional Chinese medicinal materials of "medicine and food" and "medicine diet" to form a health industry development model with national characteristics.

\subsection{Build A Talent Security System to Provide Strong Support for Health Industry Innovation}

Talent is an important part of modern market competitiveness. Therefore, it is necessary to build a complete talent guarantee system to promote the formation of a health industry product innovation chain. First, it is necessary to increase the importance of personnel training and build a professional and systematic training system. The health industry is a professional and comprehensive industry, and there is a great demand for high-quality professionals. At present, most of the employees in the health industry have not received professional skills training and systematic education. Second, broaden talent cultivation channels to further fill up the talent gap. Colleges and universities should be encouraged to develop relevant majors and cultivate high-level talents, and at the same time, actively guide social training institutions to carry out relevant training to provide high-tech talents for the health industry. Third, it is necessary to improve and improve the talent introduction mechanism of the health industry to truly retain the talents of the health industry. This not only requires government policy tilt, but also requires companies to provide talents with good working conditions. The government should actively build a policy environment that can give full play to the talents' own capabilities, solve the "worries" of talents, and provide policy support for talents; enterprises should continuously improve their own hardware infrastructure, provide conditions for scientific research and innovation, and provide reasonable salary. Work together with the government and enterprises to truly retain talents and provide intellectual guarantee for the high-quality development of the health industry.

\subsection{Improve the Construction of Traditional Infrastructure, Strengthen the Construction of New Infrastructure}

New infrastructure construction can help traditional factors of production overcome the constraints of space and time, and as an important carrier of new factors of production, promote the flow of data among various subjects, realize the comprehensive and efficient interconnection of people, machines, and things, which is the high-quality development of the health industry. The important backing. First, we must further improve the 
construction of traditional infrastructure. Accelerate the construction of social infrastructure such as roads and energy in the central and western regions, urban and rural areas, make up for the shortcomings of infrastructure, and create favorable conditions for investment promotion. Second, we must accelerate the construction of infrastructure for the elderly. China is in a period of rapid aging. The existing pension infrastructure cannot effectively meet the needs of the society in terms of quantity and quality. It is necessary to strengthen the construction of basic-level pension infrastructure and help the establishment of a community-based social pension system. Third, we must strengthen the construction of new infrastructure and promote the integrated development of new and old infrastructure. Increase the construction of new infrastructure represented by $5 \mathrm{G}$ networks and artificial intelligence, promote the in-depth integration of traditional infrastructure and new infrastructure, and actively promote the integration of big data with the health industry, especially the organic integration of technology, medical care, and elderly care. Effectively improve medical and health service capabilities in remote areas through remote technology, and provide convenience for seeking medical treatment in remote areas; use big data to establish personal health files, effectively save patients' time for medical treatment, improve protection for rational drug use, and meet the needs of personalized services and precision medicine, Inject new vitality into the development of the health industry.

\section{CONCLUSION}

The health industry includes production, education and research products and health services in all fields of maintenance, improvement, promotion and management of health. High-quality and healthy development must meet the existing and future health needs of the people. A modern health industry governance system is the foundation for achieving high-quality development of the health industry, including four aspects: market coordination, regulatory coordination, social coordination, and cultural coordination governance. At present, China's health industry has insufficient resource input, insufficient regional allocation, and industrial institutions that need to be further improved; lack of regulatory coordination, and it is urgent to improve the internal and external coordination and supervision mechanism; lack of talents leads to problems such as insufficient innovation capabilities. Therefore, it is necessary to establish an open, efficient and standardized market system; strengthen the coordination of regulatory mechanisms; strengthen health education, guide the change of social concepts, establish a modern governance system for the health industry that is governed by the government, enterprises, and society, and strengthen the construction of new infrastructure. Promote the highquality development of the health industry.

\section{ACKNOWLEDGMENTS}

Jiangxi Provincial Social Science Key Commissioned Project: Research on Jiangxi Industrial Transformation and Upgrading Based on Industry-University-Research Collaborative Innovation (No.2018WT002).

Jiangxi University of Traditional Chinese Medicine Doctoral Research Funding Project: Research on the Development of Jiangxi Traditional Chinese Medicine Cultural Tourism Industry (No.2018WT003).

Jiangxi University of Traditional Chinese Medicine College of Economics and Management Graduate Research and Training Program: Research on the effectiveness of the development policy of the Chinese medicine industry from the perspective of policy analysis.

\section{REFERENCES}

[1] Jinping Xi. At the National Health and Health Conference, Xi Jinping emphasized that people's health should be given priority in the development strategy and strive to ensure people's health in all aspects throughout the cycle. Dangjian, vol. 9, 2016, pp.4-6,9. DOI: https://kns-cnki-net443.webvpn.jxutcm.edu.cn/kcms/detail/detail.aspx $? \mathrm{dbcode}=\mathrm{CJFD} \& \mathrm{dbname}=\mathrm{CJFDLAST} 2016 \&$ filena me=DJJJ201609003\&v=WDpj6G5E4tbtEZOFbC N3eB6GPhQ0sKd\%25mmd2BXFcDdWP1d $\% 25 \mathrm{~m}$ md2B4cXS\%25mmd2Balkh11uiOgj5EPP\%25mm $\mathrm{d} 2 \mathrm{~B} \% 25 \mathrm{mmd} 2 \mathrm{~B}$

[2] Guangping Wang. Research on the Government Governance Path of the Cross-Business of the Big Health Industry. China Food \& Drug Administration Magazine, vol. 4, 2020, pp. 40-49. DOI: https://knscnki-net-

443.webvpn.jxutcm.edu.cn/kcms/detail/detail.aspx $? \mathrm{dbcode}=\mathrm{CJFD} \& \mathrm{dbname}=\mathrm{CJFDLAST} 2020 \&$ filena $\mathrm{me}=$ YPJD202004006\&v $=$ Of4TtexcCHz $\% 25 \mathrm{mmd} 2$ FYyHTBNLDIZ6IxsuuTCNQKENbayur $\% 25 \mathrm{mmd}$ 2BODOn4Uz6NYGjLeQ\%25mmd2BMMaJDKW

[3] Jiayu Zhong, Siwei Liu. Development Countermeasures of Small Tourism Towns Based on Synergetics: Taking Hunan Province as an Example. Economic Geography, vol. 32, 2012, pp. 159-164. DOI:

https://kns-cnki-net443.webvpn.jxutcm.edu.cn/kcms/detail/detail.aspx ?dbcode $=$ CJFD\&dbname $=$ CJFD2012\& filename $=J J$ DL201207027\&v=R4mkGJ95esufB1B5YCLWK5 WWR1 roc4yvp04f7RrgtZXS11hN7SUtdEyRiFKlv eDV

[4] Bida Wang, Jing Su. Can the Free Flow of Factors Lead to Regional Coordinated Development? A Theoretical Hypothesis and Empirical Test Based on "Coordinated" Agglomeration. Finance \& Trade 
Economics, vol. 41, 2020, pp. 129-143. DOI:https://kns-cnki-net-

443.webvpn.jxutcm.edu.cn/kcms/detail/detail.aspx ?dbcode $=$ CJFD\&dbname $=$ CJFDLAST2020\&filena $\mathrm{me}=\mathrm{CMJJ} 202004010 \& \mathrm{v}=\mathrm{A} 7 \mathrm{fuTlMbnk} 73 \mathrm{LhM} 176$ ppUqUVNuOsTS\%25mmd2BdgMx $558 \mathrm{c} 2 \mathrm{v} \% 25 \mathrm{~m}$ md2Bn\%25mmd2BXN9fUrHKJR1KngVpsOw0

[5] Guangping Wang, Ying Wang. Research on the Implementation Path for Supervision on Food and Drug Safety in China. Chinese Pharmaceutical Affairs, vol. 4, 2019, pp. 355-364. DOI: https://knscnki-net-

443.webvpn.jxutcm.edu.cn/kcms/detail/detail.aspx $? \mathrm{dbcode}=\mathrm{CJFD} \& \mathrm{dbname}=\mathrm{CJFDLAST} 2019 \&$ filena $\mathrm{me}=$ ZGYS201904001\&v=p6tBM $\% 25 \mathrm{mmd} 2 \mathrm{BEhQ}$ KTRpLEz06Xg9H2\%25mmd2Bo7MOm4\%25mm d2F\%25mmd2B8zy1 TLNvH6ui4LolahqmJJVtzp OwdVGS 